\title{
STABLE EQUILIBRIA UNDER THE MIGRATION MATRIX MODEL
}

\author{
M. G. BULMER \\ Department of Biomathematics, University of Oxford
}

Received 10.i.71

\section{INTRODUGTION}

THE following result has been obtained in a previous paper (Bulmer, 1971b). If a multifactorial, metric character with phenotypic variance $V$ is subject to selection for different optimal values, $\theta_{1}$ and $\theta_{2}$, in two partially isolated populations, then a stable equilibrium will exist if $\left(\theta_{1}-\theta_{2}\right)^{2} / V$ is greater than a critical value, $C$, which depends on the heritability of the character, the intensity of selection and the migration rate between the two populations. The significance of this result is that it provides a possible mechanism for the maintenance of genetic variability in natural populations.

The purpose of this paper is to exted the above result to more than two populations by using the migration matrix model introduced by Bodmer and Cavalli-Sforza (1968) for the study of random genetic drift. The general theory will be developed first, and will then be applied to a number of specific cases. The methods used in deriving the general results depend extensively on the results obtained previously for a single population and for two populations (Bulmer, 1971a, b); as in those papers, the effect of linkage disequilibrium will be ignored.

\section{The MigRATION MATRIX MODEL}

Consider a metric character, $y$, whose genetic component is determined by $\mathcal{N}$ loci with equal effects. We shall suppose that each locus has two alleles, $C_{1}$ and $C_{2}$, and that the effects of the three possible genotypes, $C_{1} C_{1}, C_{1} C_{2}$ and $C_{2} C_{2}$, are $-a, 0$ and $a$ respectively, so that there is no dominance. If the frequency of the $C_{1}$ allele at the $i$ th locus is $p_{i}$, then the genetic variance is

$$
V_{G}=2 a^{2} \sum_{i=1}^{N} p_{i} q_{i}=2 a^{2} \mathcal{N}\left(\overline{p_{i} q_{i}}\right)
$$

where $\left(\overline{p_{i} q_{i}}\right)$ is the averge value of $p_{i} q_{i}$ over the different loci. If the genetic variance is to remain approximately the same as the number of loci increases then $a^{2} \mathcal{N}$ must be approximately constant. We shall therefore regard $a^{2}$ as a quantity of order $1 / \mathcal{N}$, and we shall ignore all terms of smaller magnitude. We shall also suppose that there is an independent, normally distributed environmental component of $y$.

Suppose now that there are $n$ large populations of the same constant size, but subject to different selection pressures, and that after selection a proportion $m_{r s}$ migrates from the $r$ th to the $s$ th population. It will be assumed that the migration rates are symmetric in the sense that $m_{s r}=m_{r s}$, so that the matrix of migration rates, which will be denoted by $\mathbf{M}$, is a symmetric matrix. Under the above conditions the forward and backward migration 
matrices defined by Bodmer and Cavalli-Sforza (1968) are identical so that it is unnecessary to distinguish between them.

If the frequency of the $C_{1}$ allele at the $i$ th locus in the $r$ th population is $p_{r i}$ at the zygote stage, then the change in the gene frequency as a result of selection is, to order $a^{2}$ and hence to order $1 / \mathcal{N}$,

$$
\Delta p_{r i}=p_{r i} q_{r i}\left\{A_{r} a-\frac{1}{2} B_{r} a^{2}\left(p_{r i}-q_{r i}\right)\right\}
$$

where the coefficients $A_{r}$ and $B_{r}$ depend on the selection pressure and on the distribution of $y$ in the $r$ th population (Bulmer, 197la,b). The gene frequencies in the next generation are given by

$$
\mathbf{p}_{i}^{*}=\mathbf{M}\left(\mathbf{p}_{i}+\Delta \mathbf{p}_{i}\right)
$$

where $\mathbf{p}_{i}=\left(p_{1} i, p_{2} i, \ldots, p_{n i}\right)^{\prime}$ is the column vector of the gene frequencies at the $i$ th locus in the different populations, $\mathbf{p}_{i}^{*}$ is the corresponding column vector in the next generation, and $\Delta \mathbf{p}_{i}$ is the column vector of the $p_{r i}$ 's for $r=1,2, \ldots, n$. At equilibrium $\mathbf{p}_{i}^{*}=\mathbf{p}_{i}$, so that if $\mathbf{P}$ denotes the vector of equilibrium gene frequencies in the different populations, assumed to be the same at all loci, then

$$
\mathbf{P}=\mathbf{M}(\mathbf{P}+\Delta \mathbf{P}) .
$$

If $P_{r}$ is the equilibrium gene frequency in the $r$ th population, we shall write $P$ for the average equilibrium gene frequency, $P=\Sigma P_{r} / n$, and $\varepsilon_{r}$ for the deviation of $P_{r}$ from $P$, so that $P_{r}=P+\varepsilon_{r}$. In this paper we shall only consider the case in which $P=\frac{1}{2}$, which is considerably easier to deal with than other values of $P$, but it has previously been shown that for two populations the criterion for stability is the same for all values of $P$ in the absence of dominance (Bulmer, 1971 b). Since the $\varepsilon_{r}{ }^{\prime}$ s are of order $1 / \mathcal{N}$ (see equation 8 ) we find from equation 2 that, to order $1 / \mathcal{N}$,

$$
\Delta \mathbf{P}=\frac{1}{4} a \mathbf{A}
$$

where $\mathbf{A}$ is the column vector of the $A_{r}$ 's. From equation 4 it follows that

$$
\frac{1}{2}(\mathbf{I}-\mathbf{M}) \mathbf{1}+(\mathbf{I}-\mathbf{M}) \boldsymbol{\varepsilon}=\frac{1}{4} a \mathbf{M A}
$$

where $\mathbf{I}$ is the identity matrix, $\mathbf{I}$ is the unit vector, that is to say a column of l's, and $\varepsilon$ is the column vector of $\varepsilon_{r}$ 's. Since the rows of $\mathbf{M}$ sum to 1 the rows of $(\mathbf{I}-\mathbf{M})$ sum to zero, so that

$$
(\mathbf{I}-\mathbf{M}) \mathbf{1}=0 .
$$

Hence

$$
(\mathbf{I}-\mathbf{M}) \boldsymbol{\varepsilon}=\frac{1}{4} a \mathbf{M A} .
$$

It can also be assumed without loss of generality that $E(y)=0$ when the gene frequency is $\frac{1}{2}$ at all loci; hence the column vector of means in the different populations at equilibrium is given by

$$
\boldsymbol{\mu}=-2 \mathcal{N} a \varepsilon
$$

It will also be conveneient to write $V$ for the phenotypic variance and $h^{2} V$ for the genetic variance in a population in which the gene frequency is $\frac{1}{2}$ at all loci, so that

$$
h^{2} V=\frac{1}{2} \mathcal{N} a^{2}
$$


The actual genetic variance in the $r$ th population at equilibrium is

$$
2 P_{r} Q_{r} \mathcal{N} a^{2}=2\left(\frac{1}{2}+\varepsilon_{r}\right)\left(\frac{1}{2}-\varepsilon_{r}\right) \mathcal{N} a^{2}=\frac{1}{2} \mathcal{N} a^{2}-2 \varepsilon_{r}^{2} \mathcal{N} a^{2}
$$

which only differs from $h^{2} V$ by a quantity of order $1 / \mathcal{N}$ since $\varepsilon_{r}$ is of order $1 / \sqrt{ } \mathcal{N}$.

We must now specify more precisely the selection pressures in the different populations. It will be supposed that the fitness of an individual with phenotypic value $y$ in the $r$ th population is given by

$$
w_{r}(y)=\exp \left\{-c\left(y-\theta_{r}\right)^{2}\right\}
$$

where $c$ is a measure of the intensity of selection, assumed to be the same in all populations, and $\theta_{r}$ is the optimal value in the $r$ th population. Under this form of selection for an optimal value it can be shown that the coefficients $A_{r}$ and $B_{r}$ of equation 2 at equilibrium, that is to say when $p_{r i}=P_{r}$ at all loci, are approximately given by

$$
\begin{aligned}
& A_{r}=-k\left(\theta_{r}-\mu_{r}\right) / V \\
& B_{r}=-k / V+A_{r}^{2}
\end{aligned}
$$

where

$$
k=2 c V /(1+2 c V) \text {. }
$$

(See Bulmer, 1971 a,b.) Equation 13 would be exact if it could be assumed that the genetic variance in the $r$ th population was exactly $h^{2} V$ and that the genetic component of $y$ was exactly normally distributed. It has been shown in equation 11 that the departure from the first assumption is of order $1 / \mathcal{N}$. The departure from normality is also of order $1 / \mathcal{N}$ since the skewness and kurtosis of the genetic component of $y$ in the $r$ th population are both of order $1 / \mathcal{N}$; this can easily be shown from the standard formulae for the moments of the binomial distribution if it is remembered that $\left(P_{r}-Q_{r}\right)$ $=2 \varepsilon_{r}$ is of order $1 / \sqrt{ } \mathcal{N}$. Hence the expressions for $A_{r}$ and $B_{r}$ given by equation 13 depart from the true values by quantities of order $1 / \mathcal{N}$. They are thus sufficiently accurate since $A_{r}$ is always multiplied by $a$ and therefore need only be evaluated to order $1 / \sqrt{ } \mathcal{N}$, while $B_{r}$ is always multiplied by $a^{2}$ and need only be evaluated to order 1 . It should be noted that this argument depends on the assumption that $P=\frac{1}{2}$. If $P \neq \frac{1}{2}$ then the expressions for $A_{r}$ and $B_{r}$ given by equation 13 will depart from the true values by quantities of order $1 / \sqrt{ } \mathcal{N}$; the approximation for $B_{r}$ is still sufficiently accurate, but the approximation for $A_{r}$ is not. The ensuing complication has been dealt with in a previous paper in the case of two populations (Bulmer, 1971b), where it has been shown that the criterion for the stability of the equilibrium is unaffected. In this paper we shall only consider the simpler case in which $P=\frac{1}{2}$.

From equations $8,9,10$ and 13 we find that

$$
\left\{\left(1-h^{2} k\right)(\mathbf{I}-\mathbf{M})+h^{2} k \mathbf{I}\right\} V \mathbf{A}+k(\mathbf{I}-\mathbf{M}) \theta=\mathbf{0} .
$$

Equations 8 and 15 can be used to evaluate $\mathbf{A}$ and $\boldsymbol{\varepsilon}$ if $\boldsymbol{\theta}$ is given and if we use the additional information that $\Sigma \varepsilon_{r}=0$ by definition; it is necessary to use this information since $(\mathbf{I}-\mathbf{M})$ is a singular matrix and so does not possess an inverse. However, in the models which will be considered in this paper $\theta$ will be an "odd" vector in the sense that $\theta_{n-r}=-\theta_{r}$ for all $r$; it follows from considerations of symmetry that $\mathbf{A}$ and $\boldsymbol{\varepsilon}$ will also be "odd "vectors. 
In this case the systems of $n$ equations represented in equations 8 and 15 can be reduced to systems of $l$ equations, where $l$ is equal to $\frac{1}{2} n$ or $\frac{1}{2}(n-1)$ according as $n$ is even or odd. These equations are given by

$$
\begin{gathered}
(\mathbf{I}-\mathbf{L}) \boldsymbol{\varepsilon}^{\dagger}=\frac{1}{4} a \mathbf{L} \mathbf{A}^{\dagger} \\
\left\{\left(1-h^{2} k\right)(\mathbf{I}-\mathbf{L})+h^{2} k \mathbf{I}\right\} V \mathbf{A}^{\dagger}+k(\mathbf{I}-\mathbf{L}) \boldsymbol{\theta}^{\dagger}=\mathbf{0} .
\end{gathered}
$$

In these equations $\mathbf{A} \dagger$ and $\varepsilon \nmid$ are vectors containing the first $l A_{r}$ 's and $\varepsilon_{r}$ 's respectively, I is the $l \times l$ identity matrix, and $\mathrm{L}$ is the $l \times l$ matrix whose elements are defined as

$$
l_{r, s}=m_{r}, s-m_{r}, n-s \quad r, s=1,2, \ldots, l
$$

The matrix $(\mathbf{I}-\mathbf{L})$ will in general be non-singular and can therefore be inverted. If $\boldsymbol{\theta}$ is given, equations 16 and 17 can therefore be used directly to evaluate $\mathbf{A}$ and $\varepsilon$. (It should be noted that only $\frac{1}{2}(n-1)$ equations are required if $n$ is odd since the middle or $\frac{1}{2}(n+1)$ th values of $\varepsilon_{r}, A_{r}$ and $\theta_{r}$ must be zero.)

\section{The stability of the EQUilibrium}

To consider the stability of the equilibrium let us suppose that $p_{r i}=P_{r}$ at all loci and that the gene frequencies are then perturbed by small quantities $e_{r i}$, so that $p_{r i}=P_{r}+e_{r i}$. In the case when $P=\frac{1}{2}$ it follows from equation 2 that

$$
\Delta P_{r i}=\left(P_{r}+e_{r i}\right)\left(Q_{r}-e_{r i}\right)\left\{\left(A_{r}+\Delta A_{r}\right) a-B_{r} a^{2}\left(\varepsilon_{r}+e_{r i}\right)\right\}
$$

where $\Delta A_{r}$ is the change in $A_{r}$ due to the change in the mean caused by the perturbation, which is given by

$$
\Delta A_{r}=\left(B_{r}-A_{r}^{2}\right) 2 a \sum_{i=1}^{N} e_{r i} .
$$

(See Bulmer, 1971a,b.) If we ignore terms of smaller magnitude than $1 / \mathcal{N}$, and if we also suppose that the perturbations are sufficiently small for their squares and products to be neglected, then

$$
\Delta p_{r i}=\Delta P_{r}+\beta_{r} e_{r i}+\gamma \sum_{i=1}^{N} e_{r i}
$$

where

$$
\begin{aligned}
\beta_{r} & =-\frac{1}{4} B_{r} a^{2}-2 \varepsilon_{r} A_{r} a \\
\gamma & =\frac{1}{2}\left(B_{r}-A_{r}^{2}\right) a^{2}=-h^{2} k / \mathcal{N} .
\end{aligned}
$$

Furthermore, if $\mathbf{e}_{i}^{*}$ is the vector of deviations from the equilibrium gene frequencies at the $i$ th locus in the next generation, it follows from equations 3 and 4 that

$$
\mathbf{e}_{i}^{*}=\mathbf{M}\left\{\mathbf{e}_{i}+\left[\beta_{r} e_{r i}\right]+\gamma\left[\sum_{i} e_{r i}\right]\right\}
$$

where $\left[a_{r}\right]$ denotes a column vector whose $r$ th element is $a_{r}$. 
Let us now write

$$
\begin{aligned}
s_{r} & =\sum_{i=1}^{N} e_{r i} \\
d_{r i} & =e_{r i}-s_{r} / \mathcal{N} .
\end{aligned}
$$

Then

$$
\begin{aligned}
& \mathbf{s}^{*}=\mathbf{M}\left(1+\mathcal{N}_{\gamma}\right) \mathbf{s}+\mathbf{M}\left[\beta_{r} s_{r}\right] \\
& \mathbf{d}_{i}^{*}=\mathbf{M}\left\{\mathbf{I}+\operatorname{diag}\left(\beta_{r}\right)\right\} \mathbf{d}_{i}
\end{aligned}
$$

where diag $\left(\beta_{r}\right)$ denotes a diagnonal matrix whose $r$ th diagonal element is $\beta_{r}$. The recurrence relationship for $\mathbf{s}$ is dominated by the term $\mathbf{M}\left(1+\mathcal{N}_{\gamma}\right)$, whose dominant latent root is $\left(1+\mathcal{N}_{\gamma}\right)=\left(1-h^{2} k\right)$, since the dominant latent root of $\mathbf{M}$ is 1 ; the stability of these quantities is therefore assured. The stability of $\mathbf{d}_{i}$ depends on the dominant latent root of $\mathbf{M}+\mathbf{M} \operatorname{diag}\left(\beta_{r}\right)$, where the $\beta_{r}$ 's are of order $1 / \mathcal{N}$. Now it is shown by Fox $(1964$, p. 276) that if $\lambda$ is a latent root of a matrix $\mathbf{A}$, with left and right eigen-vectors $\mathbf{y}^{\prime}$ and $\mathbf{x}$, then the corresponding latent root of $\mathbf{A}+\delta \mathbf{A}, \delta \mathbf{A}$ being small, is $\lambda+\delta \lambda$, where

$$
\delta \lambda=\mathbf{y}^{\prime} \delta \mathbf{A x} / \mathbf{y}^{\prime} \mathbf{x}
$$

Since $\mathbf{M}$ is doubly-stochastic its largest latent root is 1 , with $\mathbf{y}=\mathbf{x}=\mathbf{1}$. Hence

$$
\delta \lambda=1^{\prime} \mathbf{M} \operatorname{diag}\left(\beta_{r}\right) \mathbf{1} / n=\sum_{r=1}^{n} \beta_{r} / n .
$$

The criterion for stability is therefore that

$$
\sum_{r=1}^{n} \beta_{r}<0
$$

To evaluate $\Sigma \beta_{r}$ we note that

$$
-\frac{1}{4} B_{r} a^{2}=\frac{\frac{1}{2} h^{2} V}{\mathcal{N}}\left(\frac{k}{V}-A_{r}^{2}\right)
$$

so that

$$
-\frac{1}{4} a^{2} \Sigma B_{r}=\frac{\frac{1}{2} h^{2} V}{\mathcal{N}}\left(\frac{n k}{V}-\Sigma A_{r}^{2}\right)=\frac{\frac{1}{2} h^{2} V}{\mathcal{N}}\left(\frac{n k}{V}-2 \mathbf{A}^{\dagger \prime} \mathbf{A}^{\dagger}\right) .
$$

Furthermore, we find from equation 16 that

$$
\begin{aligned}
-2 a \sum \varepsilon_{r} A_{r} & =-4 a \mathbf{A}^{\dagger^{\prime}} \boldsymbol{\varepsilon}^{\dagger}=-a^{2} \mathbf{A}^{\dagger \prime}(\mathbf{I}-\mathbf{L})^{-1} \mathbf{L} \mathbf{A}^{\dagger}=a^{2}\left\{\mathbf{A}^{\dagger \prime}(\mathbf{I}-\mathbf{L})^{-1}(\mathbf{I}-\mathbf{L}) \mathbf{A}^{\dagger}\right. \\
& \left.-\mathbf{A}^{\dagger \prime}(\mathbf{I}-\mathbf{L})^{-1} \mathbf{I} \mathbf{A}\right\} \\
& =\frac{2 h^{2} V}{\mathcal{N}}\left\{\mathbf{A}^{\dagger \prime} \mathbf{A}^{\dagger}-\mathbf{A}^{\dagger \prime}(\mathbf{I}-\mathbf{L})^{-1} \mathbf{A}^{\dagger}\right\} .
\end{aligned}
$$

Hence

$$
\Sigma \beta_{r}=\frac{h^{2} V}{\mathcal{N}}\left(\frac{n k}{2 V}+\mathbf{A}^{\dagger \prime} \mathbf{A}^{\dagger}-2 \mathbf{A}^{\dagger \prime}(\mathbf{I}-\mathbf{L})^{-\mathbf{1}} \mathbf{A}^{\dagger}\right)
$$


The criterion for stability is therefore that the expression in curly brackets in equation 32 should be negative, which gives the condition that

$$
\mathbf{A}^{\dagger \prime}\left\{2(\mathbf{I}-\mathbf{L})^{-1}-\mathbf{I}\right\} \mathbf{A}^{\dagger}>n k / 2 V \text {. }
$$

Evaluating $\mathbf{A}^{\dagger}$ from equation 17 and defining

$$
\mathbf{N}=(\mathbf{I}-\mathbf{L})
$$

we finally obtain the condition

$\left.\boldsymbol{\theta}^{\dagger \prime} \mathbf{N}\left\{\left(1-h^{2} k\right) \mathbf{N}+h^{2} k \mathbf{I}\right\}^{-1}\left\{2 \mathbf{N}^{-1}-\mathbf{I}\right\}\left\{1-h^{2} k\right) \mathbf{N}-h^{2} k \mathbf{I}\right\}^{-1} \mathbf{N} \boldsymbol{\theta}^{\dagger}>n V / 2 k$.

\section{The STEPPING-STONE MODEL}

The stepping-stone model has been extensively used in the study of random genetic drift (Malécot, 1962; Kimura and Weiss, 1964; Weiss and Kimura, 1965; Bodmer and Cavalli-Sforza, 1968). In the simplest form of this model it is assumed that the populations are arranged in a straight line and that migration only occurs between adjacent populations at a rate $m$. The migration matrix, $\mathbf{M}$, is therefore defined by the following equations:

$$
\begin{aligned}
& m_{11}=m_{n n}=1-m \\
& m_{r r}=1-2 m, 2 \leqq r \leqq n-1 \\
& m_{r s}=m,|r-s|=1 \\
& m_{r s}=0,|r-s|>1 .
\end{aligned}
$$

The $l \times l$ matrix $\mathbf{N}$ defined by equations 18 and 34 is equal to $m \mathbf{G}$, where $\mathbf{G}$ is defined by the following equations for $l \geqq 2$ :

$$
\begin{aligned}
& g_{11}=1 \\
& g_{l l}=\left\{\begin{array}{l}
3 \text { if } n \text { is even } \\
2 \text { if } n \text { is odd }
\end{array}\right. \\
& g_{r r}=2,2<r \leqq l-1 \\
& g_{r s}=-1,|r-s|=1 \\
& g_{r s}=0 .|r-s|>1 .
\end{aligned}
$$

If $l=1$, then $\mathbf{G}$ contains a single element, which is 2 or 1 according as $n$ is 2 or 3. If it can be assumed that both $m$ and $k$ are small, and if we write $\alpha=k / m$, then the criterion for stability contained in equation 35 can be written approximately as

$$
\boldsymbol{\theta}^{\dagger \prime} \mathbf{G}\left(\mathbf{G}+h^{2} \alpha \mathbf{I}\right)^{-1} \mathbf{G}^{-\mathbf{1}}\left(\mathbf{G}+h^{2} \alpha \mathbf{I}\right)^{-\mathbf{1}} \mathbf{G} \boldsymbol{\theta}^{\dagger}>n V / 4 \alpha .
$$

We shall consider two possible models about the distribution of the optimal values. In the first model it is assumed that the optimal value alternates between two values from one population to the next in a zig-zag manner; this may serve as a model of a mosaic pattern of variation. Under this model, which will only be defined for even values of $n$, we shall therefore suppose that

$$
\begin{aligned}
& \theta_{r}=\frac{1}{2} \theta \text { when } r \text { is even } \\
& \theta_{r}=-\frac{1}{2} \theta \text { when } r \text { is odd }
\end{aligned}
$$


so that the difference between the optimal values of two adjacent populations is $\theta$. It is easily verified that

$$
\boldsymbol{\theta}^{\dagger \prime} \mathbf{G}=\theta(1,-2,+2,-2, \ldots, \pm 2) .
$$

The criterion for stability contained in equation 38 can now be expressed in the form

$$
\frac{\theta^{2}}{V}>C
$$

where $C$ depends on the number of populations, $n$, the heritability, $h^{2}$, and the ratio $\alpha=k / m$, where $k$ is a measure of the intensity of selection and $m$ is the migration rate.

Numerical values of the stability criterion, $C$, for the zig-zag model are shown in table 1 . For $n=2 C$ is given by the simple formula

$$
C_{2}=\left(\alpha h^{2}+2\right)^{2} / \alpha \text {. }
$$

(This is in agreement with the result obtained for two populations by Bulmer, $1971 b$.) For $h^{2}=0$ this equation is valid for all values of $n$, but for $h^{2}>0$ it will be seen that $C$ tends to a limiting value for large $n$ which is equal to

\begin{tabular}{|c|c|c|c|c|c|c|c|c|}
\hline \multicolumn{9}{|c|}{$h^{2}=0$} \\
\hline$n$ & $\alpha$ & $\frac{1}{8}$ & $\frac{1}{4}$ & $\frac{1}{2}$ & 1 & 2 & 4 & 8 \\
\hline 2 & & 32 & 16 & 8 & 4 & 2 & 1 & 0.5 \\
\hline . & & . & . & . & $\cdot$ & . & . & $\cdot$ \\
\hline 32 & & 32 & 16 & 8 & 4 & 2 & 1 & 0.5 \\
\hline \multicolumn{9}{|c|}{$h^{2}=\frac{1}{2}$} \\
\hline$n$ & $\alpha$ & $\frac{1}{8}$ & $\frac{1}{4}$ & $\frac{1}{2}$ & 1 & 2 & 4 & 8 \\
\hline 2 & & 34.03 & 18.06 & $10 \cdot 12$ & 6.25 & $4 \cdot 50$ & 4.00 & $4 \cdot 50$ \\
\hline 4 & & 35.94 & 19.88 & 11.77 & $7 \cdot 61$ & $5 \cdot 44$ & 4.45 & $4 \cdot 38$ \\
\hline 8 & & 41.88 & $24 \cdot 37$ & 14.55 & 8.95 & $5 \cdot 91$ & 4.50 & $4 \cdot 18$ \\
\hline 16 & & 52.49 & $29 \cdot 11$ & $16 \cdot 32$ & 9.53 & 6.08 & 4.50 & 4.09 \\
\hline 32 & & 58.90 & 31.46 & $17 \cdot 15$ & $9 \cdot 82$ & $6 \cdot 16$ & $4 \cdot 50$ & 4.04 \\
\hline \multicolumn{9}{|c|}{$h^{2}=1$} \\
\hline$n$ & $\alpha$ & $\frac{1}{8}$ & $\frac{1}{4}$ & $\frac{1}{2}$ & 1 & 2 & 4 & 8 \\
\hline 2 & & $36 \cdot 12$ & $20 \cdot 25$ & 12.50 & 9.00 & 8.00 & $9 \cdot 00$ & 12.50 \\
\hline 4 & & 39.76 & 23.54 & $15 \cdot 21$ & 10.89 & 8.91 & $8 \cdot 76$ & $10 \cdot 62$ \\
\hline 8 & & 48.75 & $29 \cdot 11$ & 17.89 & 11.83 & $9 \cdot 00$ & 8.37 & 9.75 \\
\hline 16 & & $58 \cdot 21$ & 32.64 & 19.06 & $12 \cdot 16$ & $9 \cdot 00$ & $8 \cdot 18$ & $9 \cdot 36$ \\
\hline 32 & & 62.93 & $34 \cdot 30$ & 19.64 & $12 \cdot 33$ & $9 \cdot 00$ & 8.09 & $9 \cdot 18$ \\
\hline
\end{tabular}

TABLE 1

Values of the stability criterion, $\mathrm{C}$, for the zig-zag model

the value for $n=2$ at $\frac{1}{2} \alpha$. (This result is intuitively reasonable since each of the internal populations is exchanging migrants with its neighbours at a rate $2 m$, while the two terminal populations are exchanging migrants at a rate $m$. When $n$ is large nearly all the populations are internal, while when $n=2$ both populations are terminal; thus a migration rate of $m$ when $n=2$ 
is equivalent to a migration rate of $\frac{1}{2} m$ when $n \rightarrow \infty$.) It can be concluded that the limiting value of $C$ as $n$ tends to infinity is given by

$$
\begin{aligned}
& C_{\infty}=4 / \alpha, \quad h^{2}=0 \\
& C_{\infty}=\left(\alpha h^{2}+4\right)^{2} / 2 \alpha, \quad h^{2}>0 .
\end{aligned}
$$

There is thus a discontinuity at $h^{2}=0$ due to non-uniform convergence in this region. This means that convergence will be very slow for small values of $h^{2}$, and that for moderately large values of $n$ near $h^{2}=0 C$ is more likely to behave like equation 42 than like the second expression in equation 43 . Nevertheless, the broad generalisation can be made that the stability criterion does not vary greatly as the number of populations increases. The biological significance of the results obtained for two populations has been discussed previously (Bulmer, 1971b).

In the second model about the distribution of the optimal values, which may serve as a crude model of a geographical cline, it is assumed that the optimal value changes linearly with distance so that

$$
\theta_{r}=\theta\left\{r-\frac{1}{2}(n+1)\right\} \text {. }
$$

(This formulation ensures that $\Sigma \theta_{r}=0$.) It is easily verified that

$$
\boldsymbol{\theta}^{\dagger \prime} \mathbf{G}=\theta(1,0,0, \ldots, 0) \text {. }
$$

The criterion for stability can be expressed, as before, in the form $\theta^{2} / V>C$. Numerical values of the stability criterion, $C$, for the linear model are shown

\begin{tabular}{|c|c|c|c|c|c|c|c|c|}
\hline \multicolumn{9}{|c|}{$h^{2}=0$} \\
\hline & $\alpha$ & $\frac{1}{8}$ & $\frac{1}{4}$ & $\frac{1}{2}$ & 1 & 2 & 4 & 8 \\
\hline 2 & & 32.00 & 16.00 & 8.00 & $4 \cdot 00$ & 2.00 & 1.00 & 0.50 \\
\hline 4 & & 188 & 0.94 & 0.47 & 0.24 & $0 \cdot 12$ & 0.06 & 0.03 \\
\hline 8 & & 0.12 & 0.06 & 0.03 & 0.01 & 0.01 & 0.00 & 0.00 \\
\hline 16 & & 0.01 & 0.00 & 0.00 & 0.00 & $0 \cdot 00$ & 0.00 & 0.00 \\
\hline 32 & & 0.00 & 0.00 & 0.00 & 0.00 & 0.00 & 0.00 & 0.00 \\
\hline \multicolumn{9}{|c|}{$h^{2}=\frac{1}{2}$} \\
\hline$n$ & $\alpha$ & $\frac{1}{8}$ & $\frac{1}{4}$ & $\frac{1}{2}$ & 1 & 2 & 4 & 8 \\
\hline 2 & & 34.03 & 18.06 & $10 \cdot 12$ & $6 \cdot 25$ & 4.50 & 4.00 & 4.50 \\
\hline 4 & & $2 \cdot 31$ & 1.39 & 0.96 & 0.81 & 0.86 & $1 \cdot 14$ & 1.78 \\
\hline 8 & & 0.23 & $0 \cdot 19$ & 0.20 & 0.27 & 0.41 & $0 \cdot 70$ & 1.28 \\
\hline 16 & & 0.05 & 0.07 & $0 \cdot 10$ & 0.17 & $0 \cdot 31$ & 0.58 & $1 \cdot 12$ \\
\hline 32 & & 0.03 & 0.04 & 0.08 & $0 \cdot 14$ & 0.28 & 0.54 & 1.06 \\
\hline \multicolumn{9}{|c|}{$h^{2}=1$} \\
\hline & $\alpha$ & $\frac{1}{8}$ & $\frac{1}{4}$ & $\frac{1}{2}$ & 1 & 2 & 4 & 8 \\
\hline 2 & & $36 \cdot 12$ & $20 \cdot 25$ & $12 \cdot 50$ & 9.00 & 8.00 & $9 \cdot 00$ & 12.50 \\
\hline 4 & & 2.77 & 1.91 & 1.61 & 1.72 & $2 \cdot 28$ & 3.57 & 6.22 \\
\hline 8 & & 0.39 & 0.41 & 0.53 & $0 \cdot 82$ & 1.41 & 2.57 & $4 \cdot 87$ \\
\hline 16 & & $0 \cdot 13$ & 0.20 & 0.34 & 0.62 & $1 \cdot 17$ & 2.25 & $4 \cdot 39$ \\
\hline 32 & & 0.09 & 0.15 & 0.29 & 0.55 & 1.08 & $2 \cdot 12$ & $4 \cdot 19$ \\
\hline
\end{tabular}
in table 2. For $n=2$ the linear model and the zig-zag model are of course

\section{TABLE 2}

Values of the stability criterion, $\mathrm{C}$, for the linear model

identical, so that equation 42 is valid. As $n$ tends to infinity it is clear that $C$ tends to a limiting value given by

$$
C_{\infty}=\frac{1}{2} h^{4} \alpha .
$$


Thus for large values of $n$ stability is easier to achieve the smaller the value of $\alpha$, that is to say the smaller the selection intensity compared with the migration rate. It must be remembered, however, that this result depends on the assumption that the migration rate, $m$, as well as the selection intensity, $k$, is small. It is not worth while to try to relax this assumption since the simple form of the stepping-stone model used here, which assumes that migration only occurs between neighbouring populations, is itself only realistic if the migration rate is small. In the next section we shall construct a more realistic model of a continuous geographic cline which assumes that migration takes place in continuous space.

\section{A MODEL IN GONTINUOUS SPAGE}

As a continuous analogue of the migration matrix model we shall suppose that after selection an individual moves a random distance $u$ in one dimension, with density function $f(u)$ which is symmetrical about zero with variance $\sigma^{2}$. In order to avoid the problem of edge effects we shall suppose that the species is distributed continuously around a great or small circle on the surface of the world. In practice, of course, a species will usuaily only occupy a segment of a circle, but it makes the mathematical treatment much easier to suppose that the species occupies the whole circle and it seems unlikely that this idealisation will introduce a serious distortion. It can also be assumed in the case of a one-dimensional cline that the species is distributed on a circle rather than on a band of appreciable width since only movement in the direction of the cline need be considered; it must therefore be understood that the density function, $f(u)$, refers only to movement in the direction of the cline. We shall denote the position of an animal on the circle by its angular distance, $x$, measured in radians from an arbitrary fixed origin on the circle; it must therefore be assumed that $u$ is also measured in radians.

If $\theta(x)$ is the optimal value at $x$, then $\theta(x)$ must clearly be a periodic function so that $\theta(x+2 \pi)=\theta(x)$. The simplest function which satisfies this requirement is

$$
\theta(x)=T \cos p x
$$

where $p$ is an integer. (This formulation ensures that

$$
\int_{0}^{2^{\pi}} \theta(x) d x=0 .
$$

It also assumes that the origin has been fixed in such a way that $\theta(x)$ takes its maximum value, $T$, when $x=0$; this can always be done since the origin is arbitrary, and it is therefore unnecessary to introduce a phase-shift parameter into the formulation.) It will often be appropriate to assume that $p=2$ since this makes the optimal values the same on opposite sides of the world; for example, in the case of a North-South cline going round a meridian of longitude it would be reasonable to suppose that the optimal value depends on the latitude regardless of the hemisphere in which the point lies. For the moment, however, we shall not assign a specific value to $p$.

In the same way that the optimal values, $\theta_{r}$, of the migration matrix model have been replaced by a continuous function, $\theta(x)$, so must the constants $A_{r}, B_{r}, \varepsilon_{r}$ and so on be replaced by continuous functions $A(x), B(x)$, 
$\varepsilon(x)$ and so on; it is clear that these functions must also be periodic so that, for example, $A(x+2 \pi)=A(x)$. Since the migration matrix, $\mathbf{M}$, has been replaced by the density function, $f(u)$, the analogue of equation 8 is

$$
\int_{-\infty}^{+\infty}\left\{\varepsilon(x+u)-\varepsilon(x)+\frac{1}{4} a A(x+u)\right\} f(u) d u=0 .
$$

If we take Taylor expansions of $\varepsilon(x+u)$ and $A(x+u)$ in powers of $u$ about $x$ and if we remember that $E(u)=E\left(u^{3}\right)=0, E\left(u^{2}\right)=\sigma^{2}$, we find that, to order $u^{3}$,

$$
\frac{1}{2} \sigma^{2} \varepsilon^{\prime \prime}(x)+\frac{1}{4} a A(x)+\frac{1}{8} a \sigma^{2} A^{\prime \prime}(x)=0 .
$$

If we write $\mu(x)=-2 \mathcal{N} a \varepsilon(x)$ by analogy with equation 9 , then the analogue of equation 13 is

$$
\begin{aligned}
& A(x)=-k\{\mathcal{T} \cos p x+2 \mathcal{N} a \varepsilon(x)\} / V \\
& B(x)=-k / V+A^{2}(x)
\end{aligned}
$$

so that

$$
A^{\prime \prime}(x)=-k\left\{-p^{2} T \cos p x+2 \mathcal{N} a \varepsilon^{\prime \prime}(x)\right\} / V .
$$

Substituting for $\varepsilon^{\prime \prime}(x)$ from equation 51 in equation 49 we find that

$$
\sigma^{2}\left(1-h^{2} k\right) A^{\prime \prime}(x)-2 h^{2} k A(x)=p^{2} k T \sigma^{2} \cos (p x) / V .
$$

A particular solution of equation 52 is

$$
A(x)=\frac{-p^{2} k \mathcal{T}^{2} \cos p x}{V\left\{p^{2}\left(1-h^{2} k\right) \sigma^{2}+2 h^{2} k\right\}} .
$$

This is the required solution since the solution of the homogeneous equation involves only the functions cosh and sinh which are aperiodic. Substituting this expression for $A(x)$ in equation 49 we find that the required solution for $\varepsilon(x)$ is

$$
\varepsilon(x)=\frac{-\frac{1}{2} a k T\left(1-\frac{1}{2} p^{2} \sigma^{2}\right) \cos p x}{V\left\{p^{2}\left(1-h^{2} k\right) \sigma^{2}+2 h^{2} k\right\}} .
$$

Finally, the analogue of the stability criterion $\Sigma \beta_{r}<0$ (see equation 28) is

$$
\int_{0}^{2^{\pi}} \beta(x) d x<0
$$

where

$$
\begin{aligned}
\beta(x) & =-\frac{1}{4} a^{2} B(x)-2 a \varepsilon(x) A(x) \\
& =\frac{h^{2} V}{2 \mathcal{N}}\left\{\frac{k}{V}-A^{2}(x)-\frac{8 \varepsilon(x) A(x)}{a}\right\}
\end{aligned}
$$

Hence

$$
\int_{0}^{2^{\pi}} \beta(x) d x=\frac{h^{2} V \pi}{2 \mathcal{N}}\left[\frac{2 k}{V}-\frac{p^{2} k^{2} T^{2} \sigma^{2}\left(4-p^{2} \sigma^{2}\right)}{V^{2}\left\{p^{2}\left(1-h^{2} k\right) \sigma^{2}+2 h^{2} k\right\}^{2}}\right]
$$

since

$$
\int_{0}^{2 \pi} \cos ^{2} p x d x=\int_{0}^{2 \pi} \cos ^{2} x d x=\pi
$$


The criterion for stability can therefore be written

$$
\frac{p^{2} T^{2}}{V}>\frac{2\left\{p^{2}\left(1-h^{2} k\right) \sigma^{2}+2 h^{2} k\right\}^{2}}{k \sigma^{2}\left(4-p^{2} \sigma^{2}\right)} .
$$

Since the unit of measurement of the migration law is the radius of the circie, which is approximately 4000 miles if it is a great circle and which will in any case be large, $\sigma^{2}$, which is in square units, will be extremely small, and so the criterion for stability is approximately given by

$$
\frac{p^{2} T^{2}}{V}>2 h^{4} k / \sigma^{2}
$$

Let us now see how this result can be interpreted in practice. We first consider a North-South cline in a species which extends over tropical and temperate latitudes. It is reasonable to suppose, for reasons discussed earlier, that $p=2$, so that $T$ can be estimated from the difference between the optimal values on the Equator and at $45^{\circ}$ North or South. Let us suppose that the mean value of the character is 50 on the Equator and 100 at $54^{\circ}$, so that $T$ can be estimated as $100-50=50$, and that the phenotypic variance is 10 ; then $p^{2} T^{2} / V=4 \times 50^{2} / 10=1000$. Let us also suppose that $k=0 \cdot 01$, and that the standard deviation of the migration law (counting only movement in a North-South direction) is 4 miles, which is equivalent to onethousandth of the earth's radius, so that $\sigma^{2}=10^{-6}$. The criterion for stability is that

$$
10^{3}>2 h^{4} \times 10^{4}
$$

or

$$
h^{2}<1 / \sqrt{20}=0 \cdot 22 .
$$

It can be concluded that under these conditions a heritability of 0.22 can be maintained by the existence of the cline.

In the case of a more limited geographical cline, which may not be in a North-South direction, it is preferable to estimate $T$ from the observed slope of the cline, which we will denote by $\lambda$. The absolute value of the theoretical slope is given by

$$
\left|\theta^{\prime}(x)\right|=p T|\sin p x| .
$$

Now $|\sin p x|$ takes its maximum value of 1 when $p x=\frac{1}{2} \pi$ and its minimum value of 0 when $p x=0$. In general we do not know $x$, since we do not know where the cline, if extended round the world, would have its maximum and minimum values; it therefore seems reasonable to assume that we are halfway between the positions where the cline has its maximum and minimum slope, that is to say that $p x=\frac{1}{4} \pi$, so that $|\sin p x|=1 / \sqrt{ } 2$. We shall therefore assume that

$$
\lambda=T p / \sqrt{ } 2
$$

so that the criterion for stability can be expressed as

$$
\frac{\lambda^{2}}{V}>h^{4} k / \sigma^{2}
$$


Since $\lambda$ is the slope of the cline per unit distance, both sides of this inequality have the same dimensions of distance, so that distance can be measured in any units and need not be measured in radians. It is interesting to observe that equation 65 is equivalent to the criterion

$$
\frac{\theta^{2}}{V}>h^{4} k / 2 m
$$

for the linear stepping stone model (see equation 46) if we take the unit of distance as the distance between two adjacent populations so that $\theta$ and $\lambda$ have the same meaning and so that the variance of the migration distance is $2 m$. A similar analogy between the two models has been observed by Malécot (1962) in connection with the effect of migration on the coefficient of kinship.

\section{Summary}

1. The migration matrix model of Bodmer and Cavalli-Sforza (1968) is used to construct a model for the behaviour of a metric character subject to selection for different optimal values in different populations with migration between them.

2. A criterion for the stability of an equilibrium position is derived, and is applied firstly to the stepping-stone model under two hypotheses about the distribution of the optimal values in the different populations, and then to a model of a geographic cline in continuous space.

3. The maintenance of genetic variability by this means in natural populations is discussed.

\section{REFERENGES}

BODMER, W. F., AND GAVALLI-SFORZA, L. L. 1968. A migration matrix model for the study of random genetic drift. Genetics, 59, 565-592.

BULMER, M. G. 1971a. The stability of equilibria under selection. Heredity, 27, 157-162.

BULmer, M. G. 1971b. Stable equilibria under the two island model. Heredity, 27, 321-330. Fox, L. 1964. An Introduction to Numerical Linear Algebra. Clarendon Press, Oxford.

KIMURA, M., AND WEISS, G. H. 1964. The stepping stone model of population structure and the decrease of genetic correlation with distance. Genetics, 49, 561-576.

MALÉCOT, C. 1962. Migration et parenté génétique moyenne. Entretiens de Monaco en sciences humaines, 205-212. (Reprinted in Malécot, 1966.).

MALÉCOT, G. 1966. Probabilités et Herédité. (Cahier 47 de l'Institut national d'études démographiques.) Presses Universitaires de France, Paris.

WEISS, G. H., AND KIMURA, M. 1965. A mathematical analysis of the stepping stone model of genetic correlation. J. Appl. Prob., 2, 129-149. 